\title{
Epidemiology of Foot-and-Mouth Disease outbreaks in Thailand from 2011 to 2018
}

\author{
Thanicha Chanchaidechachai ${ }^{1}$, Helmut Saatkamp ${ }^{1}$, Mart de Jong${ }^{1}$, Chaidate Inchaisri ${ }^{2}$, \\ Henk Hogeveen ${ }^{1}$, and Bart H. P. Borne ${ }^{1}$ \\ ${ }^{1}$ Wageningen University \& Research \\ ${ }^{2}$ Chulalongkorn University Faculty of Veterinary Science
}

November 1, 2021

\begin{abstract}
Foot-and-Mouth Disease (FMD) is one of the most important animal diseases hindering livestock production in Thailand. In this study, a temporal and spatial analysis at the subdistrict level was performed on FMD outbreak reports in Thailand from 2011 to 2018. Risk factors associated with FMD outbreaks were investigated using generalised estimating equations. The results showed that the incidence of FMD outbreaks was the highest in 2016 and was affected by season, with a peak in FMD outbreaks occurring in the rainy-winter season. The occurrences of FMD were mostly distributed as a number of small clusters within a few subdistricts. Some high-risk areas with repetitive outbreaks were detected in the central regions. Risk factors significantly associated with FMD outbreaks included the size of the dairy, beef, and pig populations in each subdistrict, the distance to the nearest subdistrict with an outbreak, the percentage of forest area, international border contact, the presence of a livestock market, and the occurrence of an FMD outbreak in a neighbouring subdistrict in the previous month. This study helped to identify high-risk areas and periods of FMD outbreaks in Thailand. Together with the identified risk factors, its results can be used to optimise the FMD control program in Thailand and in other countries having a similar livestock industry and FMD situation.
\end{abstract}

\section{Hosted file}

20-10-2021Epidemiology of Foot-and-Mouth Disease outbreaks in Thailand .docx available at https://authorea.com/users/444070/articles/543891-epidemiology-of-foot-and-mouthdisease-outbreaks-in-thailand-from-2011-to-2018 